\title{
Competência dos discentes de graduação em nutrição sobre a avaliação subjetiva global
}

\author{
Competence of undergraduate nutrition students on global subjective assessment
}

Competencia de los discentes de graduación en nutrición sobre la evaluación subjetiva global

\begin{abstract}
Fábio Costa de Vasconcelos ${ }^{1 *}$, Lizomar de Jesus Maués Pereira Moia ${ }^{1,2}$, Irland Barroncas Gonzaga Martens ${ }^{3}$, Cinthia Regina Sales Furtado Vieira ${ }^{1}$
\end{abstract}

\section{RESUMO}

Objetivo: Avaliar os conhecimentos dos discentes do curso de nutrição sobre a avaliação subjetiva global. Método: Estudo descritivo, analítico-exploratório e transversal, com abordagem qualitativa. A amostra foi de cinquenta e três discentes, regularmente matriculados, ambos os sexos, cursando o último semestre de quatro Instituições de Ensino Superior do curso de Nutrição. Os dados foram coletados por meio de entrevista aberta e semiestruturada, cujo roteiro foi composto por perguntas abertas relacionadas ao processo teórico e prático, assim como conhecimento, habilidades e prática sobre avaliação subjetiva global, após a aprovação do Comitê de Ética e Pesquisa. Os dados qualitativos foram analisados pela técnica análise de conteúdo, modalidade temática. Resultados: De acordo com os temas recorrentes e críticos na pesquisa as habilidades e competências específicas para realizar diagnóstico pela Avaliação Subjetiva Global, foram limitadas por falta de treinamento, conhecimento técnico e aulas práticas insuficientes. Conclusões: Os discentes são conscientes da importância de realizar a avaliação nutricional criteriosa para o diagnóstico nutricional adequado. Porém, relatam abordagens distintas de assuntos na teoria e prática, ou seja, não estão integradas. A maioria relatou insegurança em diagnosticar o paciente devido ausência ou aulas práticas insuficientes, comprometendo a competência dos discentes.

Palavras-Chave: Estudantes, Ensino, Avaliação Nutricional, Competência Profissional.

\begin{abstract}
Objective: To evaluate the knowledge of the students of the course of nutrition on the global subjective evaluation. Method: Descriptive, analytical-exploratory and cross-sectional study with a qualitative approach. The sample was fifty-three students, regularly enrolled, both sexes, attending the last semester of four Institutions of Higher Education of the Nutrition course. The data were collected through an open and semistructured interview, whose script was composed of open questions related to the theoretical and practical process, as well as knowledge, skills and practice on global subjective assessment, after approval of the Ethics and Research Committee. The qualitative data were analyzed by the technique of content analysis, thematic modality. Results: According to recurrent and critical themes in the research the specific skills and

1 Universidade do Estado do Pará (UEPA), Belém-Pará. * E-mail: fcvnutri@yahoo.com.br

2 Professora Dra. Adjunto do Mestrado em Ensino em Saúde na Amazônia Universidade do Estado do Pará.

${ }^{3}$ Professora Dra. Associada da Universidade Federal do Pará.
\end{abstract}


competences to perform diagnosis by Global Subjective Assessment were limited due to lack of training, technical knowledge and insufficient practical classes. Conclusions: The students are aware of the importance of performing the nutritional assessment carefully for the appropriate nutritional diagnosis. However, they report different approaches to issues in theory and practice, that is, they are not integrated. Most reported insecurity in diagnosing the patient due to absence or insufficient practical classes, compromising the competence of the students.

Key words: Students, Teaching, Nutrition Assessment, Professional Competence.

\section{RESUMEN}

Objetivo: Evaluar los conocimientos de los alumnos del curso de nutrición sobre la evaluación subjetiva global. Método: Estudio descriptivo, analítico-exploratorio y transversal, con abordaje cualitativo. La muestra fue de cincuenta y tres discentes, regularmente matriculados, ambos sexos, cursando el último semestre de cuatro Instituciones de Enseñanza Superior del curso de Nutrición. Los datos fueron recolectados por medio de una entrevista abierta y semiestructurada, cuyo guion fue compuesto por preguntas abiertas relacionadas al proceso teórico y práctico, así como conocimiento, habilidades y práctica sobre evaluación subjetiva global, tras la aprobación del Comité de Ética e Investigación. Los datos cualitativos fueron analizados por la técnica análisis de contenido, modalidad temática. Resultados: De acuerdo con los temas recurrentes y críticos en la investigación, las habilidades y competencias específicas para realizar diagnóstico por la Evaluación Subjetiva Global, fueron limitadas por falta de entrenamiento, conocimiento técnico y clases prácticas insuficientes. Conclusiones: Los estudiantes son conscientes de la importancia de realizar la evaluación nutricional adecuada para el diagnóstico nutricional adecuado. Sin embargo, relatan enfoques distintos de asuntos en la teoría y práctica, sea, no están integradas. La mayoría relató inseguridad en diagnosticar al paciente debido ausencia o clases prácticas insuficientes, comprometiendo la competencia de los estudiantes.

Palabras clave: Estudiantes, Enseñanza, Evaluación Nutricional, Competencia Profesional.

\section{INTRODUÇÃO}

O profissional de saúde tem o compromisso de ser inovador e ético, e que seja apto a desenvolver ações de prevenção, promoção, proteção e reabilitação da saúde individual e coletiva. É imprescindível que haja coerência entre a formação e as exigências esperadas de atuação profissional e a necessidade de democratização da participação e dos acessos da sociedade aos direitos a educação e saúde (TREVISO P e COSTA BEP, 2017)

A formação é, sobretudo, a condição de refazer permanentemente as relações profissionais com os usuários de modo responsável e comprometido. Atualmente as mudanças na formação profissional está associada aos eixos científicos, tecnológicos, sociais, econômicos, político, cultural e epidemiológicos (FILHO JRF, et al., 2017).

Neste contexto, as novas Diretrizes Curriculares Nacionais (BRASIL, 2001) do curso de graduação em nutrição, definem o perfil do nutricionista em formação, isto é, deverá ser um profissional generalista, humanista e crítico, capaz de atuar em todas as áreas do conhecimento em que alimentação e nutrição estejam envolvidas (COSTA EQ, et al., 2013; JUNQUEIRA TS e COTTA RMM, 2014).

Sendo assim, as Instituições de Ensino Superior (IES) deverão formar profissionais capacitados nas diversas áreas de atuação, entre elas, a nutrição clínica, baseada nas competências e habilidades específicas afim de nortear o acadêmico durante o processo de ensino e aprendizagem. Além de adquirir competência 
técnica, o discente deverá compreender, analisar e intervir os problemas inerentes a clínica, tendo como referência protocolos de avaliação, intervenção nutricional, e monitoramento do estado clínico nutricional do paciente hospitalizado, a fim de viabilizar assistência nutricional, para sistematizar, padronizar e aperfeiçoar os serviços na área de atuação (JUNQUEIRA TS e COTTA RMM, 2014).

Referente a avaliação nutricional, destaca-se a avaliação subjetiva do estado nutricional de indivíduos enfermos, idealmente, deve ser feita por meio de abordagem multivariada e, essencialmente clínica. Assim, a Avaliação Subjetiva Global (ASG), padronizada por Detsky AS et al. (1987), é vista como um dos melhores métodos para obtenção do diagnóstico do paciente e padrão ouro pela American Society for Parenteral and Enteral Nutrition (HANUSCH, FD, et al., 2016; SANTOS AF, et al., 2017).

A ASG não é apenas utilizada para classificar o estado nutricional, mas também para identificar pacientes que apresentam maior risco de sofrer complicações associadas ao estado nutricional durante sua internação. Logo a ferramenta é utilizada tanto para o diagnóstico adequado da desnutrição quanto para o prognóstico, assim ajuda a orientar as intervenções adequadas (JENSEN GL, et al., 2012; SOUSA JUNIOR JB, et al., 2016).

Caberá ao discente ter competência e habilidade para realizar avaliação do estado nutricional do paciente, com base em protocolos pré-estabelecidos, como a ASG; além de identificar risco, deficiências e o diagnóstico nutricional para realizar o tratamento dietoterápico ou terapia nutricional de forma adequada.

Assim, conforme o exposto, surgiram os seguintes questionamentos: o processo de diagnóstico nutricional a partir da avaliação subjetiva está sendo contemplado na formação acadêmica? O discente tem a percepção da finalidade de realizar avaliação subjetiva para fins de diagnóstico nutricional? E por fim, os discentes do curso de nutrição apresentam conhecimentos, competências, habilidades e atitudes na aplicabilidade da ASG?

Estas questões norteadoras situam a presente pesquisa como uma investigação que tem seu núcleo nos significados apreendidos a partir dos participantes, e teve como objetivo avaliar os conhecimentos dos discentes do curso de graduação em nutrição sobre a avaliação subjetiva global.

\section{MÉTODO}

Trata-se de um estudo descritivo, analítico-exploratório, transversal e de abordagem qualitativa. Foram adotados os seguintes critérios de inclusão: discentes de ambos os sexos, regularmente matriculados no $8^{\circ}$ semestre do curso de graduação em nutrição de quatro Instituições de Ensino Superior (IES), de Belém (PA). Foram excluídos discentes irregulares quanto a matrícula, os que estavam cursando os demais semestres de graduação e aqueles que não concordaram em assinar o Termo de Consentimento Livre e Esclarecido (TCLE).

Destaca-se que, por serem concluintes devem apresentar aptidão para realizar a avaliação subjetiva global, uma vez que, é uma habilidade específica inerente a avaliação nutricional inserida na matriz curricular dos cursos de graduação em nutrição. Além disso, enfatiza-se que as instituições de ensino superior selecionadas foram todas que já apresentavam discentes concluintes do curso de nutrição.

De um universo de 100 discentes do 8 ำ período das quatro IES, a amostra foi constituída de 53 alunos. Para análise qualitativa, o número amostral foi considerado suficiente quando houve reincidência de informações, considerando relatos ímpares de cada um dos discentes entrevistados (MINAYO MCS, 2014). As quatro IES autorizaram a execução da pesquisa, após ciência dos objetivos e metodologia.

Os dados foram coletados por meio de entrevista aberta e semiestruturada, cujo roteiro foi composto por perguntas abertas em que se procura apreender questões relacionadas ao processo teórico e prático, assim 
como conhecimento, habilidades e prática na avaliação nutricional subjetiva, a seguir: Como você percebe a relação teoria e prática em sua formação? Em sua opinião quais os conhecimentos, habilidades e atitudes necessárias para a realização da ASG? Relate se você tem segurança em dar o diagnóstico nutricional do paciente pela ASG?

A entrevista foi gravada e teve duração média de 20 minutos. Segundo Minayo MCS (2014), a entrevista aberta e semiestruturada tem a vantagem da elasticidade quanto a duração, permitindo uma profundidade em relação ao tema analisado. Bem como, a interação entre o entrevistador e entrevistado favorecendo as respostas espontâneas. O conteúdo gravado foi transcrito na íntegra e, fez-se uma leitura exaustiva do material, com a finalidade de apropriação de conteúdos recorrentes, críticos ou contraditórios, seguindo o modelo para tratamento, redução e análise, conforme preconizado pela literatura.

Os dados foram submetidos a análise de conteúdo, modalidade temática, o qual abrangem três etapas: pré-análise, etapa de organização dos dados, que tem como finalidade sistematizar e operacionalizar as ideias iniciais a fim de conduzir desenvolvimento de operações sucessivas; exploração do material, consiste no processo de codificação, decomposição ou enumeração dos relatos ou textos; e por fim, o tratamento dos resultados, inferência e interpretação (BARDIN L, 2011).

O tratamento dos resultados, a partir da análise dos pontos norteadores usados na elaboração das questões da entrevista, permitiram elencar temas que deram origem a formação de quatro categorias temáticas: (1) Visão geral do componente curricular teórico e prático; (2) Habilidades e competência em relação a avaliação subjetiva global e (3) Desafios para realizar avaliação subjetiva para fins de diagnóstico nutricional.

O estudo foi aprovado pelo Comitê de Ética em Pesquisa com seres humanos do Conselho Nacional de Saúde, sob o número do CAAE: 78795617.1.0000.5169 e parecer número 2.363.116; garantindo os aspectos éticos pautados segundo os preceitos da Declaração de Helsinque e do Código de Nuremberg e respeitando as normas de pesquisa envolvendo seres humanos do Conselho Nacional de Saúde (Resolução CNS № 466/2012).

A entrevista foi realizada em local reservado na IES, durante o período de intervalo de aula. O sigilo dos participantes foi garantido por meio da utilização do sistema alfanumérico, isto é, foram codificados pela letra D (discente), seguidos dos números dados aos questionários (D01), e as quatro Instituições de Ensino Superior pelas letras A, B, C, D.

\section{RESULTADOS E DISCUSSÃO}

A pesquisa foi composta por 53 discentes, distribuídos da seguinte forma de acordo com a IES: 14 (IES A); 16 (IES B); 10 (IES C) e 13 (IES D). A média de idade dos participantes foi de 25,49 $\pm 7,12$ anos; e conforme o gênero, verifica-se que $90,56 \%$ eram do sexo feminino.

Os resultados da pesquisa foram confrontados com o referencial teórico sobre ensino em saúde, habilidades e competências na área clínica e estratégias para o ensino no curso de nutrição. De acordo com a interpretação dos dados, apropriou-se dos conteúdos recorrentes, crítico ou contraditórios, destacando-se as seguintes categorias temáticas:

\section{Visão geral do componente curricular teórico e prático}

Conforme Pereira IDL e Lages I (2013) e Siqueira-Batista R et al. (2013), a educação é centrada em competências e está associada à uma aprendizagem baseada nas atitudes, habilidades e conhecimentos, sendo fundamental para que os estudantes atuem de maneira responsável, criativa, ativa e reflexiva na construção de sua vida acadêmica, pessoal, social e, em paralelo, profissional. 
Logo, um projeto pedagógico pautado por princípios filosóficos e psicopedagógicos explícitos são necessários para a definição das competências e habilidades, das estratégias educacionais e metodológicas, dos objetivos de aprendizagem a serem alcançados, e por fim, do sistema de avaliação da aprendizagem adquirida na teoria e prática (COSTA EQ, et al., 2013).

Através da análise, observar-se que os discentes de duas IES, compreendem que há necessidade de ter um processo de ensino aprendizagem pautado na teoria e prática, como se verifica nos relatos recorrentes:

"[...] no decorrer dos anos deveria ter tido mais aulas práticas...deveria unir teoria e prática... algumas disciplinas que deveriam ter mais práticas, teve muita teoria...". A-D05.

"[...] muitas vezes não estão conexas, a gente vê uma coisa e na realidade é outra e muitas vezes não é o que utilizamos na prática...". A-D08.

“[...] nem sempre a prática está de acordo com a teoria, temos que aprender a lidar com situações adversas...". B-D04.

"[...] teoria excelente, só a prática que deixou muito a desejar, desmerecer. Avaliação nutricional deveria ter o foco maior e foi muito a desmerecer, faltou mais treinamento...". B-D05.

Pode-se extrair das falas dos discentes que a teoria é fundamental, porém a pratica é que possibilitará ter habilidades na área de atuação, entre elas a avaliação nutricional; outros enfatizam como aspecto central pouco tempo de aulas práticas, sendo desenvolvidas no período final do curso, que abrange o período de estágios curriculares.

A aprendizagem está baseada na relação entre a teoria e a prática. Sem a compreensão e conhecimento adequado do assunto teórico, não haverá rendimento de exercer a prática em sua plenitude. As adversidades durante a prática podem ocorrer e muitas vezes os discentes não estão preparados para enfrenta-las, podendo ocorrer constrangimento, dúvidas, erros de interpretação e diagnósticos inadequados (BRIGHENTI J, et al., 2015).

De acordo com os relatos dos discentes das IES C e D, destacam-se que os assuntos abordados na teoria estarão interligados com o saber-fazer, abrangendo o conhecimento operacional e procedimental, a seguir:

"[...] interligadas, uma depende da outra, teoria antes da prática, mas devem ser casadas, pois possibilita o aluno a compreender melhor o que ele aprendeu na teoria...". C-D02.

"[...] as aulas práticas contribuem muito mais que a teórica, a prática acaba exercendo o que você vai fazer no seu futuro profissional, acaba ajudando mais os alunos do que na teórica...". C-D09.

"[...] eu percebi algo muito produtivo e esclarecedor. Consegui absorver bastante informação e as práticas foram muito importantes, nos deixou aptos para desenvolver as atividades...". D-D04.

"[...] a relação entre a teoria e a prática na formação profissional é de extrema importância para auxiliar 0 aluno adquirir experiência nas atividades inerentes aos profissionais de nutrição...". DD09.

Compreende-se que, é extremamente importante que o docente tenha domínio teórico no assunto a ser abordado e que transmita segurança, técnicas e habilidades aos discentes. Mas para isso, caberá ao professor ser o facilitador da aprendizagem e verificar quais são as metodologias mais eficazes para cada situação, buscando o raciocínio reflexivo e crítico. O saber dos professores é construído ao longo de uma carreira profissional, sendo assim, tais conhecimentos não são adquiridos apenas por um conjunto de 
conteúdos cognitivos imutáveis e tradicionais. O processo contínuo de aprendizagem prática e teórica permite que o mestre aprenda gradualmente o saber (BRIGHENTI J, et al., 2015).

Neste contexto, são determinantes que as metodologias de ensino e o processo de ensino aprendizagem estejam centradas no aluno, em diferentes cenários, níveis hierárquicos de atenção e nas diversas áreas de atuação, contemplado a necessidade de reformular a orientação profissional, baseada na teoria e prática desde o início do curso, permeando toda a formação do futuro nutricionista (TREVISO P e COSTA BEP, 2017).

\section{Competência e habilidades em relação a avaliação subjetiva global}

As competências e habilidades exercem uma grande importância no processo ensino aprendizagem, permitindo a capacidade de contextualizar demandas complexas de um determinado tema e assunto, mobilizando aspectos psicossociais cognitivos ou não cognitivos, além de integrar saberes essenciais na forma de planejar e executar os conhecimentos adquiridos (COSTA EQ, et al., 2013; JUNQUEIRA TS e COTTA RMM, 2014).

Além da competência técnica o profissional deverá estar capacitado para conhecer, compreender, analisar, interpretar e intervir nas diversas situações da área da nutrição clínica, entre elas a avaliação e diagnóstico nutricional de pacientes, a fim de estar preparado para o mercado de trabalho, buscando sempre a valorização, a ética e a humanização (COSTA EQ, et al., 2013).

A competência não está alinhada apenas com o conhecimento técnico, como realizar anamnese nutricional e exame físico, mas também com capacidade de integração, comunicação, raciocínio, humanização, capacidade reflexiva, atualização, e por fim habilidades e atitudes necessárias para que os estudantes e profissionais atuem de maneira ativa, responsável e criativa na área de nutrição clínica (SIQUEIRA-BATISTA R, et al., 2013; COSTA EQ, et al., 2013; JUNQUEIRA TS e COTTA RMM, 2014).

Ser competente dá respaldo ao nutricionista de adaptar-se em situações diversas nas áreas de atuação, e de lidar com a imensa quantidade de informações. Para isso, a formação acadêmica deverá ser baseada em uma prática contextualizada e interdisciplinar, onde o discente desempenha um papel de sujeito, com a incorporação de fatores que fazem parte do ser unitário, ou seja, afetivos, sociais e cognitivos. Tendo a capacidade de encontrar recursos e respostas criativas e eficazes para problemas inéditos que se apresentam (COSTA EQ, et al., 2013; JUNQUEIRA TS e COTTA RMM, 2014).

Dessa forma, o docente deverá compatibilizar os conteúdos e temas trabalhados na área clínica de acordo com as necessidades, fragilidades, aspirações e expectativas dos discentes; buscar o significado educativo, social e epistemológico do conhecimento que transmite ou faz seus discentes aprenderem. Para que o processo de ensino aprendizagem ocorra de forma integrada e alcance seus objetivos metodológicos a partir da relação objetivo-conteúdo (BASSOLI F, 2014).

No entanto, o professor terá que ter a consciência da responsabilidade pela formação de futuros profissionais (nutricionista clínicos) e a busca pelo espaço institucional voltado para a construção de uma identidade coletiva de ser professor; e neste contexto, para que tenha a possibilidade de continuar aprendendo e melhorando a abordagem didático-pedagógica utilizada, mediante o uso de metodologias ativas, as IES deverão valorizar o docente e utilizar estratégias na busca por formação continuada, e aperfeiçoamento na área pedagógica. Assim, o professor deve ter um enfoque no ensino reflexivo e crítico, a fim de desafiar, instigar, contribuir e estimular os discentes na construção de habilidades e competências que fortaleçam o compromisso profissional (BASSOLI F, 2014; TREVISO P e COSTA BEP, 2017).

Nessa abordagem recorrente, os estudantes das IES A e C compreendem que a competência e habilidade está associada com o conhecimento teórico e aplicabilidade na prática da avaliação subjetiva global. A construção da habilidade em perceber as anormalidades nutricionais inerentes ao paciente, através da

REAS/EJCH | Vol. 11 (3) | e231 | DOI: https://doi.org/10.25248/reas.e231.2019 Página 6 de 9 
percepção e inspeção de alterações clínicas e nutricionais são enfatizados para a realização da avaliação. Pontos relevantes relatados foram o "olhar clínico", abordagem ao paciente e o enfoque no treinamento para executar a avaliação subjetiva global, conforme demostrado a seguir:

“[...] olhar clínico, perceber visualmente como ele está debilitado ou não, fazer inspeção de tudo aquilo que pode ser risco nutricional..." A-D01.

"[...] conhecimento teórico, inspeção, observação e avaliação crítica, com a prática ajuda na atividade..." A-D10.

"[...] olhar clínico, perceber como o paciente está visualmente debilitado ou não e fazer uma inspeção para associar aos riscos nutricionais...". C-D01.

"[...] pessoa não treinada não dá diagnóstico, pode induzir ao paciente em relação as respostas...". C-D06.

"[...] acompanhar o paciente tem que ter habilidade. No estágio tivemos muita prática com a avaliação subjetiva global..." C-D08

Pode-se extrair relatos contraditórios dos discentes das IES B e D, que para se obter o estado nutricional do paciente pela ASG é necessário o uso de equipamentos, como adipômetro e fita métrica. Não relatam a competência técnica correta a ser realizada para obter o diagnóstico. Há ênfase de técnicas objetivas, questionário de consumo alimentar e conhecimento de várias doenças para realizar a avaliação, que é caracterizada pela subjetividade, conforme demonstrado a seguir:

"[...] habilidades com aparelho para medir pregas...saber como entrevistar de forma simplificada, temos que perguntar de forma que o paciente possa entender...". B-D04

"[...] saber usar dados antropométricos, usar adipômetro e fita métrica...". B-D05

“...tem que ter conhecimento do histórico da pessoa, fazer o recordatório, conhecer várias doenças, dados antropométricos, dados bioquímicos...". B-D06

“[...] para a realização da ASG é necessário ter as técnicas de aferimento das medidas antropométricas, o conhecimento da população estudada...". D-D06

É um método simples, de custo muito baixo, com boa reprodutibilidade e confiabilidade. $O$ desenvolvimento desse instrumento fundamenta-se em uma abordagem essencialmente clínica em forma de questionário, abordando a história clínica e exame físico do paciente (SOUSA JUNIOR JB, et al., 2016).

Por outro lado, a precisão diagnóstica depende da capacidade do investigador em buscar informações precisas, detalhadas e que lhe sirvam de substrato para alcançar o diagnóstico nutricional, ou seja, a técnica é sujeita ao viés do entrevistador (GOMES ES e SALOMON ALR, 2014; CALAZANS FDCF, 2015).

\section{Desafios para realizar avaliação subjetiva global para fins de diagnóstico nutricional}

O diagnóstico nutricional de um indivíduo enfermo é obtido por métodos objetivos e subjetivos. Este último, tem como instrumento principal a avaliação subjetiva global, que valoriza essencialmente a progressão de perda de peso do doente e o período em que ocorreu, além de outras variáveis clínicas significantes como a existência de alterações do apetite, a presença de sintomas gastrointestinais e as mudanças da capacidade funcional (OLIVEIRA TR e FORTES RC, 2015).

De posse de informações obtidas pela anamnese clínica e exame físico o paciente será classificado como: A - bem nutrido; B - moderadamente desnutrido ou com suspeita de desnutrição, e C - gravemente 
desnutrido. A ASG foi desenhada a obter poucos resultados falso-positivos, isto é, o paciente classificado como desnutrido grave tem poucas chances de ser nutrido ou moderadamente desnutrido. Logo, o questionário o torna um teste de alta especificidade. Portanto, o diagnóstico nutricional correto do paciente condiz em uma conduta nutricional adequada pelo nutricionista (HANUSCH FD, et al. 2016).

Nesta abordagem, extraem-se relatos críticos de insegurança para realizar avaliação subjetiva para fins de diagnóstico nutricional, dos discentes das IES A, B e D, a seguir:

\section{"[...] não tenho tanta segurança, pois depende da gravidade do paciente...". A-D03.}

"[...] nem tanto, depende dos pacientes que for realizar a ASG, em alguns pacientes fica difícil, não ter essa prática gera dúvidas...". A-D05.

“[...] hoje não por falta de prática e pouca vivência...”. B-D02.

"[...] não tenho segurança, faltou essa prática e exercício. Tanto que quando terminou o estágio questionamos com a preceptora que teve muito pouco tempo de estágio...". B-D08.

"[...] ainda não realizei nenhuma avaliação de forma autônoma, somente duas vezes sob supervisão de uma nutricionista, não tenho segurança em dar o diagnóstico...". D-D04

\section{“[...] não tenho segurança em realizar ASG...". D-D08}

A ausência ou aulas práticas insuficientes proporcionam aos alunos um aprendizado mais inconsistente e um grande desafio na formação acadêmica. A necessidade do desenvolvimento da relação professor-alunopaciente durante o período de práticas clínicas foi confirmada nas respostas dos discentes entrevistados, desencadeando a insegurança no diagnóstico nutricional pela ASG. Esta realidade poderá trazer consequências graves no processo de intervenção nutricional do paciente.

As aulas práticas de avaliação nutricional têm a finalidade de despertar e manter os discentes envolvidos no processo de investigação científica, desenvolver habilidades técnicas de diagnóstico e ter a capacidade de solucionar problemas, compreender e interpretar conceitos específicos na área nutricional. Neste sentido, a aprendizagem, baseada na prática clínica, deve garantir um currículo com múltiplas oportunidades, que propiciem a integração docente-discente no ensino, na pesquisa e na extensão (TREVISO P e COSTA BEP, 2017).

A síntese dos resultados desta pesquisa permitiu observar que os Saberes devem integrar a construção do processo de ensino aprendizagem pactuadas com as IES - Discente - Docente: o Saber-conhecer, está relacionado com a compreensão e interpretação das questões abordadas na ASG; o Saber-fazer, garantir aos discentes as habilidades cognitivas para aplicar a ASG a partir da realização da prática de forma contínua a nível hospitalar; o Saber-ser, propõe-se ao discente ser um profissional ético e moral, mantendo uma relação discente $\mathrm{x}$ paciente $\mathrm{x}$ docente pautada nos valores, princípios e atitudes profissionais válidas nas áreas de atuação de forma ética e moral; o Saber-conviver, estabelecer uma abordagem clara e compreensiva da ferramenta que será aplicada ao paciente, que o mesmo tire todas as dúvidas sobre o procedimento, mantendo sempre os direitos e o respeito aos mesmos; e por fim, o Saber utilizar, que corresponde ao aperfeiçoamento das competências a partir dos conhecimentos adquiridos (TREVISO P e COSTA BEP, 2017).

\section{CONCLUSÃO}

A pesquisa mostrou que os discentes são conscientes da importância de realizar a avaliação nutricional criteriosa para obtenção do diagnóstico nutricional adequado. Porém, relatam abordagens distintas de assuntos na teoria e prática, ou seja, não estão integradas. Destacam, também, que a técnica para obter diagnóstico nutricional do paciente pela avaliação subjetiva global requer sensibilidade, observação e 
inspeção. Mas, de forma errônea, alguns citam que instrumentos são necessários para o diagnóstico. A maioria relatou insegurança em diagnosticar o paciente devido ausência ou aulas práticas insuficientes, comprometendo a competência dos discentes.

\section{REFERÊNCIAS}

1. BARDIN L. Análise de conteúdo. 70 ed. São Paulo; 2011; 280p.

2. BASSOLI, F. Atividades práticas e o ensino-aprendizagem de ciência (s): mitos, tendências e distorções. Ciênc. Educ, 2014; 20(3): 579-593.

3. BRASIL. Ministério da Educação. Conselho Nacional de Educação. Câmara de Educação Superior. Institui diretrizes curriculares nacionais do curso de graduação em nutrição. Resolução CNE/CES nำ, de 7 de novembro de 2001. Diário Oficial da União. Brasília, 2011. $39 \mathrm{p}$.

4. BRIGHENTI J, BIAVATTI VT, SOUZA TR. Metodologias de Ensino-Aprendizagem: uma abordagem sob a percepção dos alunos. Revista GUAL, 2015; 8(3): 281-304.

5. CALAZANS FDCF, GUANDALINI VR, PETARLI GB et al. Triagem Nutricional em Pacientes Cirúrgicos de um Hospital Universitário de Vitória, ES, Brasil. Nutr. Clín. Diet. Hosp., 2015; 35(3): 34-41.

6. COSTA EQ, DOMINGUES JR, MALHEIROS LR et al. Desafios à reforma curricular em um curso de graduação em nutrição. Demetra, 2013; 8(3): 469-485.

7. DETSKY AS, MCLAUGHLIN JR, BAKER JP et al. What is subjective global assessment of nutritional status? JPEN J Parenter Enteral Nutr, 1987; 11(1): 8-13.

8. FILHO JRF, COSTA MV, FORSTER AC et al. New national curricula guidelines that support the use of interprofessional education in the Brazilian context: An analysis of key documents. J Interprof Care, 2017; 754-760.

9. GOMES ES, SALOMON ALR. Métodos subjetivos utilizados para identificar o perfil nutricional de pacientes portadores de neoplasia: Avaliação Subjetiva Global Produzida pelo Paciente (ASG-PPP) e Avaliação Subjetiva Global (ASG). Revisa, 2014; 2: 115-124.

10. HANUSCH FD, SILVA MDGB, PRADO LVDS et al. Avaliação nutricional de pacientes submetidos à cirurgia do trato gastrointestinal: associação entre avaliação subjetiva global, ferramentas de triagem nutricional e métodos objetivos. Nutr. Clín. Diet. Hosp., 2016; 36(2): 10-19.

11. JENSEN GL, HSIAO PY, WHEELER D. Adult nutrition assessment tutorial. JPEN J Parenter Enteral Nutr. 2012; 36(3): 267-74.

12. JUNQUEIRA TS, COTTA RMM. Matriz de ações de alimentação e nutrição na Atenção Básica de Saúde: referencial para a formação do nutricionista no contexto da educação por competências. Ciênc. Saúde coletiva. 2014; 19(5): 1459-1474.

13. MINAYO MCS. O desafio do conhecimento: pesquisa qualitativa em saúde. 14. ed. São Paulo: Hucitec. 2014; 408p.

14. OLIVEIRA TR, FORTES RC. Prevalência de Desnutrição em Pacientes Cirúrgicos em Terapia Nutricional e sua relação com os Parâmetros Objetivos e Subjetivos de Avaliação Nutricional. Com. Ciências Saúde. 2015; 26(3/4): 115-126.

15. PEREIRA IDF, LAGES I. Diretrizes curriculares para a formação de profissionais de saúde: competências ou práxis? Trab. educ. saúde, 2013; 11(2): 319-338.

16. SANTOS AFD, RABELO JUNIOR AA, CAMPOS FLB et al. Scored patient-generated Subjective Global Assessment: Length of hospital stay and mortality in cancer patients. Rev. Nutri., Campinas, 2017; 30(5): 545-553.

17. SIQUEIRA-BATISTA R, GOMES AP, ALBUQUERQUE VS et al. Educação e competências para o SUS: é possível pensar alternativas à(s) lógica(s) do capitalismo tardio? Cien Saude Colet, 2013; 18(1): 159-170.

18. SOUSA JUNIOR JB, CASTRO T, LIMA L et al. Comparação entre avaliação subjetiva global e o novo diagnóstico nutricional proposto pela ASPEN em pacientes cirúrgicos. BRASPEN J, 2016; 31(4): 305-310.

19. TREVISO P, COSTA BEP. Percepção de profissionais da área da saúde sobre a formação em sua atividade docente. Texto Contexto Enferm, 2017; 26(1): 01-09. 